\title{
Serum vitamin D deficiency and risk of epithelial ovarian cancer in Lagos, Nigeria
}

\author{
Emmanuel Adekunle Sajo ${ }^{1}$, Kehinde Sharafadeen Okunade ${ }^{1,2}$, Gbenga Olorunfemi ${ }^{3}$, Kabiru Afolarin Rabiu ${ }^{4}$ and Rose Ihuoma Anorlu ${ }^{1,2}$ \\ ${ }^{1}$ Department of Obstetrics and Gynaecology, Lagos University Teaching Hospital LUTH, PMB 12003, Idi-Araba, Lagos, Nigeria \\ ${ }^{2}$ Department of Obstetrics and Gynaecology, Faculty of Clinical Sciences, College of Medicine, University of Lagos, PMB 12003, Idi-Araba, Lagos, Nigeria \\ ${ }^{3}$ Division of Epidemiology and Biostatistics, School of Public Health, University of the Witwatersrand, Parktown 2193, Johannesburg, South Africa \\ ${ }^{4}$ Department of Obstetrics and Gynaecology, Lagos State University College of Medicine, PMB 21266, Ikeja, Lagos, Nigeria
}

\section{Abstract}

The studies that have evaluated the association between vitamin $D$ and risk of ovarian cancer have reported inconsistent findings. Many of these studies were carried out in regions with relatively low sunshine all year round unlike in Africa. This study was aimed to determine the relationship between vitamin $\mathrm{D}$ deficiency and epithelial ovarian cancer (EOC) amongst women in Lagos, Nigeria. We conducted a case-control study involving women with histologically confirmed EOC (case group) and an equal number of healthy women without cancer (control group) treated at the gynaecological oncology units of two public tertiary hospitals in Lagos, Nigeria, between 1 August, 2016 and 31 May, 2017. Relevant information was obtained from the participants using a structured interviewer-administered questionnaire, and then, venous blood samples were collected and analysed for serum 25-hydroxyvitamin D levels using the CALBIOTECH ${ }^{\circledR} 25(\mathrm{OH})$ vitamin D ELISA kit. The descriptive statistics were conducted for all relevant data, and the multivariable analysis using binary logistic regression model was performed to examine the association between vitamin D deficiency and EOC after adjusting for all possible confounders. The mean age of the participants was $50.6 \pm 11.1$ years. There was no statistically significant association between serum vitamin D deficiency and EOC ( $p=0.09$ ). However, $10 \mathrm{mmol} / \mathrm{L}$ change in circulating vitamin D levels was associated with EOC amongst the study participants (adjusted odds ratio 0.96; 95\% confidence interval 0.930.99; $p=0.04$ ), but following adjustment for potential confounders in a multivariable analysis, there was no statistically significant relationship observed with EOC (adjusted odds ratio 0.99; 95\% confidence interval 0.97-1.00; $p=0.06$ ). In addition, there was no evidence of an interaction effect between these confounders and change in circulating $25(\mathrm{OH}) \mathrm{D}$ levels in relation to the risk of EOC. The study revealed no statistically significant association between the circulating levels of vitamin D and the risk of EOC. A better assessment of sun exposure in the future as well as better dietary compositional data may help to clarify whether the association between vitamin D and EOC actually exists. Therefore, the future large prospective longitudinal studies are recommended to further examine this relationship and then evaluate the possible need for vitamin D supplementation in women with an increased risk of EOC in Nigeria.

Keywords: CALBIOTECH®, EOC, Lagos, Nigeria, ovarian cancer, vitamin D

Correspondence to: Kehinde S Okunade Email: sokunade@unilag.edu.ng

ecancer 2020, 14:1078

https://doi.org/10.3332/ecancer.2020.1078

Published: $23 / 07 / 2020$

Received: 31/03/2020

Publication costs for this article were supported by ecancer (UK Charity number 1176307).

Copyright: (C) the authors; licensee ecancermedicalscience. This is an Open Access article distributed under the terms of the Creative Commons Attribution License (http:// creativecommons.org/licenses/by/3.0), which permits unrestricted use, distribution, and reproduction in any medium, provided the original work is properly cited. 


\section{Introduction}

Ovarian cancer is a very deadly cancer, and it is the second most common cause of gynaecological cancer death in Nigeria [1]. Over 70\% of ovarian cancer patients present at the late stage of the disease when little or nothing could be done, thereby resulting in poor prognosis and survival [2]. In Nigeria, epithelial ovarian cancer (EOC) accounts for 60\%-68\% of all histological types of ovarian cancer [2-4]. Unlike the most prevalent cancer, cervical cancer, which has preventive and screening strategies, there has not been any preventive modality nor validated screening programme for ovarian cancer as most of the risk factors are not modifiable [2].

Ovarian cancer incidence is now rising in Nigeria and most low- and middle-income countries due to the gradual transition to a westernised way of life [5], and this will impact significantly on the already overstretched healthcare system in these counties. Several hereditary/genetic, reproductive, nutritional and environmental risk factors have been directly or indirectly linked to the development of ovarian cancer. Risk estimates are mostly based on a family history with a lifetime risk estimate of 2-5 times the population risk for individuals who have one first degree relative with ovarian cancer [6,7]. Reproductive factors such as early age at menarche and late age at menopause, which increase the risk of ovarian cancer, have also been shown to modestly increase the risk of ovarian cancer [8]. Several protective factors have been established in the previous studies, and these include increasing parity and breastfeeding [9], oral contraceptives [10] and increased physical activities [11]. The process of cellular inflammation, wound healing and hormonal influence are all thought to be involved in the poorly understood ovarian cancer pathogenesis [12].

In the medical world, there is an increased interest in the diverse functions of vitamin D in the human body [13, 14]. Vitamin D, a fat-soluble vitamin, which is mostly produced from sunlight exposure $[15,16]$, has been found in experimental studies to inhibit the growth of ovarian cancer cells and also stimulate programmed cell death in similar cells. The circulating form of vitamin D, 25-hydroxyvitamin D (25[OH]D), is converted to 1,25-dihydroxy vitamin $\mathrm{D}\left(1,25[\mathrm{OH}]_{2} \mathrm{D}\right.$ by 1-a-hydroxylase enzyme which is ubiquitous in most tissues of the body. Both normal and cancerous ovarian cells have vitamin D receptors, through which the active form of vitamin $D$ acts as a paracrine hormone to cause antiproliferative action through the non-calcaemic pathway. It probably reduces the risk of cancers by the induction of tumour suppressor genes such as p53, p21 and DNA-mismatched repair genes [17].

There are no reliable data on the intake of vitamin D-fortified diet amongst Nigerians, and it is difficult to study the dietary intake of vitamin $\mathrm{D}$ as it is strongly associated with lactose intake $[18,19]$. Thus, circulating vitamin $\mathrm{D}$ levels may be a useful alternative in investigating a potential relationship with ovarian cancer. However, to the best of authors' knowledge, no study has examined the association between low circulating vitamin D levels and the risk of ovarian cancer in Sub-Saharan African women. The studies that evaluated this association reported inconsistent findings [17, 20,21], and many of these studies were conducted in regions with a predominantly low prevalence of micronutrient deficiencies, unlike what is obtained in most of the resource-constraint settings including Nigeria. This study, therefore, aimed to determine the association between serum vitamin D deficiency and the risk of EOC amongst women in Lagos, Nigeria. The finding of this study may give an insight into the future preventive role of this important vitamin against this extremely lethal female gynaecological cancer.

\section{Materials and methods}

\section{Study design and setting}

This was a case-control study carried out at the gynaecological wards of the Lagos University Teaching Hospital (LUTH) and Lagos State University Teaching Hospital (LASUTH) between August 1, 2016 and May 31, 2017. LUTH and LASUTH are the two main public tertiary institutions that offer specialised cancer care in Lagos, Nigeria. Lagos State is located in the Southwestern part of Nigeria, Coordinates: $6^{\circ} 35^{\prime} \mathrm{N} 3^{\circ} 45^{\prime} \mathrm{E}$; Land Area-3,474 km² (1,341 sq. $\left.\mathrm{mL}\right)$.

\section{Study population and eligibility criteria}

The study enrolled two groups of women by consecutive sampling technique. The case group included women who had staging laparotomy and histologic confirmation of epithelial ovarian cancer (EOC), whereas their control group comprised of age-matched ( \pm 5 years) women 
attending the gynaecology outpatient clinics of the two hospitals on account of infertility and had no clinical or ultrasound scan evidence of ovarian lesion. Women who used vitamin D-containing mineral supplements in the past 3 months before enrollment and those who refused consent at enrolment or withdrew their consents in the course of the study were excluded from the study.

\section{Sample size calculation}

The sample size was determined by applying the formula for a comparison of two means using data from the published study by Webb et al [22]. With a standard deviation of $18.7 \mathrm{nmol} / \mathrm{l}$ and between-group vitamin $\mathrm{D}$ level mean difference of $15 \mathrm{nmol} / \mathrm{L}$, a power analysis revealed that a minimum sample size of 35 women in each group (total sample size of 70 women) is required to ensure at least $80 \%$ power for detecting the anticipated between-group differences and to compensate for a non-response rate of $10 \%$. We enrolled 35 women with histologically confirmed EOC and 35 cancer-free comparison groups into the study.

\section{Data collection}

A structured interviewer-administered questionnaire was used to collect data such as sociodemographic characteristics, medical history, family history of cancer, smoking as well as information on the use of vitamin D supplements and dressing style (whether covered or uncovered). The covered dressing was defined as the covering of the entire body except for the hands and feet, whereas the uncovered dressing was the common dressing with exposure of the face, neck, hand and feet. The participant's skin colour was also recorded as evident from the face, neck, arms and legs. The anthropometric measurements such as weight and height were determined, and the body mass index (BMI) was calculated for each participant. A venous blood sample $(4 \mathrm{~mL})$ was collected from each participant and sent to the laboratory. The samples were then centrifuged, and the serum was stored at $-20^{\circ} \mathrm{C}$ until analysis. The level of total 25 -hydroxyvitamin $D$ in each serum sample was measured by using a solid-phase competitive enzyme-linked immunosorbent assay (ELISA) as per the manufacturer's instructions (Calbiotech ${ }^{\circledR}$, Spring Valley, CA, USA). The specificity of the assay for measurement of total $25(\mathrm{OH})$ vitamin D was $100 \%$ with an intraassay variation of $<6 \%$ [23]. Low serum vitamin $\mathrm{D}$ was defined as a level below $75 \mathrm{nmol} / \mathrm{L}$ [15].

\section{Statistical analysis}

The statistical analysis was conducted using Stata Version 16 Statistical Package (Stata I/C, StataCorp LP, Texas, USA). The descriptive statistics were computed for all relevant data. Categorical variables were described as frequency and percentages. Normally distributed continuous variables were described as mean \pm standard deviation, whereas non-normally distributed continuous variable was described as median and interquartile range. The Kolmogorov-Smirnov test was used to test for normality. The independent sample $t$-test or Mann-Whitney U-test was used to compare the continuous variables amongst participants with ovarian cancer and those without ovarian cancer. The Pearson's Chi-square test (or Fischer's exact test) was used to test the association between categorical variables and EOC. The univariable and multivariable conditional logistic regression modellings were conducted. The model building utilised variables based on the authors' clinical knowledge and biological plausibility. The potential confounders of EOC and circulating vitamin D levels, specifically participants' age, parity, menopausal status, body mass index, family history of cancer, educational qualification, skin complexion and coexisting medical morbidity were considered in the logistic regression models. The participants' age and variables with $p$-value $<0.20$ such as a family history of cancer and skin colour were built into the final multivariable conditional logistic regression model using vitamin $D$ as a continuous primary explanatory variable. Subsequently, we estimated the main and circulating vitamin D level covariate interaction effects on the risk of EOC. A twotailed test of hypothesis was assumed, and the $p$-value $<0.05$ was considered to be statistically significant.

\section{Ethical considerations}

An ethical approval for the study was obtained from the Health Research Ethics Committee of the two participating teaching hospitals before the recruitment of participants. All participants were counselled before enrolment and read and signed an informed consent form. The investigators ensured strict confidentiality of participants' information. 


\section{Results}

The characteristics of the study women in each group are shown in Table 1. The mean age was $50.46 \pm 11.68$ years for women with epithelial ovarian cancer $(E O C)$ and $50.40 \pm 11.10$ years for those without cancer $(p=0.47)$. There were no statistically significant differences in the parity $(p=0.83)$, occupation ( $p=0.99)$, educational level $(p=0.36)$, menopausal status $(p=0.63)$, BMI $(p=0.73)$, skin colour $(p=0.37)$, alcoholic beverage ingestion $(p=0.61)$ and family history of cancer $(p=0.04)$ between the EOC group and their cancer-free comparison group. The overall prevalence of vitamin D deficiency was $77.5 \%(n=54)$ with $85.7 \%(n=30)$ of women with EOC having low serum vitamin $D$ level compared to $68.5 \%(n=24)$ of women without EOC $(p=0.09)$. The median (interquartile range) level of vitamin D was 33.8 (23.0-53.0 $\mathrm{nmol} / \mathrm{L})$ in the EOC group and 50.0 (32.5-93.0 nmol/L) in the cancer-free group ( $p<0.01)$ (Fig. 1).

The most common histological type of EOC in the study was serous cystadenocarcinoma (80.0\%), whereas the least common was malignant Brenner tumour (2.9\%). Majority of the women had elevated serum cancer antigen $125(91.4 \%)$ and presented with an advanced stage of the disease (94.3\%) (Table 2).

Table 3 shows no statistically significant association between the categories of vitamin D ( $<75 \mathrm{nmol} / \mathrm{L}$ versus $\geq 75 \mathrm{nmol} / \mathrm{L}$ ) and EOC (adjusted odds ratio 2.75; 95\% confidence interval 0.84-9.00; $p=0.09$ ). However, there was a statistically significant association between $10 \mathrm{mmol} / \mathrm{L}$ change in circulating serum vitamin D levels and EOC after a univariable analysis (adjusted odds ratio 0.96; $95 \%$ confidence interval 0.93$0.99 ; p=0.04$ ). With further multivariable conditional logistic regression analysis after adjusting for the potential major risk factors of EOC such as age, family history of cancer and skin colour, the change in circulating serum vitamin D levels was found to have no statistically significant association with EOC (adjusted odds ratio 0.99; 95\% confidence interval 0.97-1.00; $p=0.06$ ). However, there was an almost 6-fold statistically significant association between the patients' family history of cancer and the risk of EOC (adjusted odds ratio 5.88; 95\% confidence interval 1.05-32.94; $p=0.04$ ). In addition, there was no evidence of an interaction effect between age, family history of cancer or skin colour and circulating 25(OH)D levels in relation to the risk of EOC (Table 4).

\section{Discussion}

This study showed no association between circulating vitamin D levels and epithelial ovarian cancer (EOC). Initial support for a protective role for vitamin $D$ in ovarian cancer was provided by previous ecological studies that recorded inverse associations between ultraviolet-B (UV-B) irradiance exposure from sunlight and ovarian cancer incidence or mortality rates [24-27]. However, a few recent studies that evaluated this association further have reported conflicting findings [17, 20,21]. This study reported similar findings to the studies conducted by Tworoger et al [20], Arslan et al [17] and Zheng et al [28], where no significant associations were detected between circulating serum 25-hydroxyvitamin D levels and the risk of ovarian cancer. However, it appears that Arslan et al [17] did not find any significant difference in the median serum 25-hydroxyvitamin D levels between the case group with EOC and their healthy comparison group. On the contrary to the finding, Bakhru et al [21] in a similar study conducted in Michigan, USA, a non-tropical country in North America, reported a significant association between low vitamin D levels and ovarian cancer. Bakhru et al [21] reported that participants with ovarian cancer had a 3-fold increased risk of having low levels of vitamin D when compared to their counterparts without ovarian cancer. The findings by Toriola et al [29] also suggested an increased risk of ovarian cancer in women with low-to-insufficient serum 25(OH)D concentration, thus suggesting that healthy women are usually exposed to sunlight, the major source of naturally occurring vitamin D, all year round [30, 31] as they are mobile and outgoing compared to their counterparts with ovarian cancer who are usually docile and tend to stay indoors all day as a result of their illness. However, the absence of an association between low circulating serum vitamin $D$ levels reported in this present study might reflect the predominantly high prevalence of nutritional deficiencies in this region, but this will require further validation in a more robust longitudinal study that will also objectively assess the diets of these women.

We also recorded in this study that women with a family history of any form of cancer had about 6-fold increased odds of EOC compared to women with no family history of cancer. This was similar to the findings by Tworoger [20] in Boston, Massachusetts, in 2007 and Zheng et al in Nashville, Tennessee, in 2010 [29]. This further suggests that there is a genetic link in the evolution of ovarian cancer as was previously described for women with the BRCA gene mutation [32]. 
Table 1. Baseline characteristics of participants ${ }^{a}$.

\begin{tabular}{|c|c|c|c|}
\hline \multirow{2}{*}{ Characteristics } & \multicolumn{2}{|c|}{ Epithelial ovarian cancer } & \multirow{2}{*}{$p$-value } \\
\hline & Yes, $n=35$ (\%) & No, $n=35$ (\%) & \\
\hline \multicolumn{3}{|l|}{ Age in years } & \multirow{4}{*}{0.47} \\
\hline$<50$ & $15(42.9)$ & $18(51.4)$ & \\
\hline$\geq 50$ & $20(57.1)$ & $17(48.6)$ & \\
\hline Mean age $\pm S D$ & $50.40 \pm 11.68$ & $50.40 \pm 11.10$ & \\
\hline \multicolumn{3}{|l|}{ Parity } & \multirow{5}{*}{0.83} \\
\hline 0 & $6(17.1)$ & $5(14.3)$ & \\
\hline $1-4$ & $22(62.9)$ & $21(60.1)$ & \\
\hline$>4$ & $7(20.0)$ & $9(25.7)$ & \\
\hline Median parity (IQR) & $3(1-4)$ & $3(1-5)$ & \\
\hline \multicolumn{3}{|l|}{ Occupation } & \multirow{4}{*}{0.99} \\
\hline Civil servant/retiree & $12(34.3)$ & $11(31.4)$ & \\
\hline Caterer/farmer/housewife & $5(14.3)$ & $6(17.1)$ & \\
\hline Trading & $18(51.4)$ & $18(51.4)$ & \\
\hline \multicolumn{3}{|l|}{ Educational level } & \multirow{3}{*}{0.36} \\
\hline None/primary & $8(22.9)$ & $5(14.3)$ & \\
\hline Secondary/tertiary & $27(77.1)$ & $30(85.7)$ & \\
\hline \multicolumn{3}{|l|}{ Menopausal state } & \multirow{3}{*}{0.63} \\
\hline Premenopausal & $13(37.1)$ & $15(42.9)$ & \\
\hline Postmenopausal & $22(62.9)$ & $20(57.1)$ & \\
\hline \multicolumn{3}{|l|}{$\mathrm{BMI}$ in kg/m² } & \multirow{5}{*}{0.73} \\
\hline 18.5-24.9 (normal) & $14(40.0)$ & $11(31.4)$ & \\
\hline 25.0-29.9 (overweight) & $13(37.1)$ & $14(40.0)$ & \\
\hline$\geq 30$ (obese) & $8(22.9)$ & $10(28.6)$ & \\
\hline Median BMI (IQR) & $26.0(22.7-29.2)$ & $27.5(23.4-32.4)$ & \\
\hline \multicolumn{3}{|l|}{ Skin complexion } & \multirow{4}{*}{0.37} \\
\hline Black & $7(20.0)$ & $7(20.0)$ & \\
\hline Dark brown & $16(45.7)$ & $21(60.0)$ & \\
\hline Light & $12(34.3)$ & $7(20.0)$ & \\
\hline \multicolumn{3}{|l|}{ Alcohol intake } & \multirow{3}{*}{$0.61^{b}$} \\
\hline No & $32(91.4)$ & $34(97.1)$ & \\
\hline Yes & $3(8.6)$ & $1(2.9)$ & \\
\hline \multicolumn{3}{|l|}{ Family history of cancer } & \\
\hline No & $27(77.1)$ & $33(94.3)$ & 0.04 \\
\hline Yes & $8(22.9)$ & $1(5.7)$ & \\
\hline \multicolumn{3}{|l|}{ Vitamin D categories } & \multirow{3}{*}{0.09} \\
\hline Normal ( $\geq 75 \mathrm{nmol} / \mathrm{L})$ & $5(14.3)$ & $11(31.5)$ & \\
\hline Deficiency $(<75 \mathrm{nmol} / \mathrm{L})$ & $30(85.7)$ & $24(68.5)$ & \\
\hline
\end{tabular}

Abbreviations: BMI, body mass index (calculated as weight in kilograms divided by the square of height in metres).

${ }^{a}$ Values are given as mean \pm SD, median (interquartile range) or number (percentage) unless stated otherwise.

${ }^{\mathrm{b}}$ Fisher's exact test. 


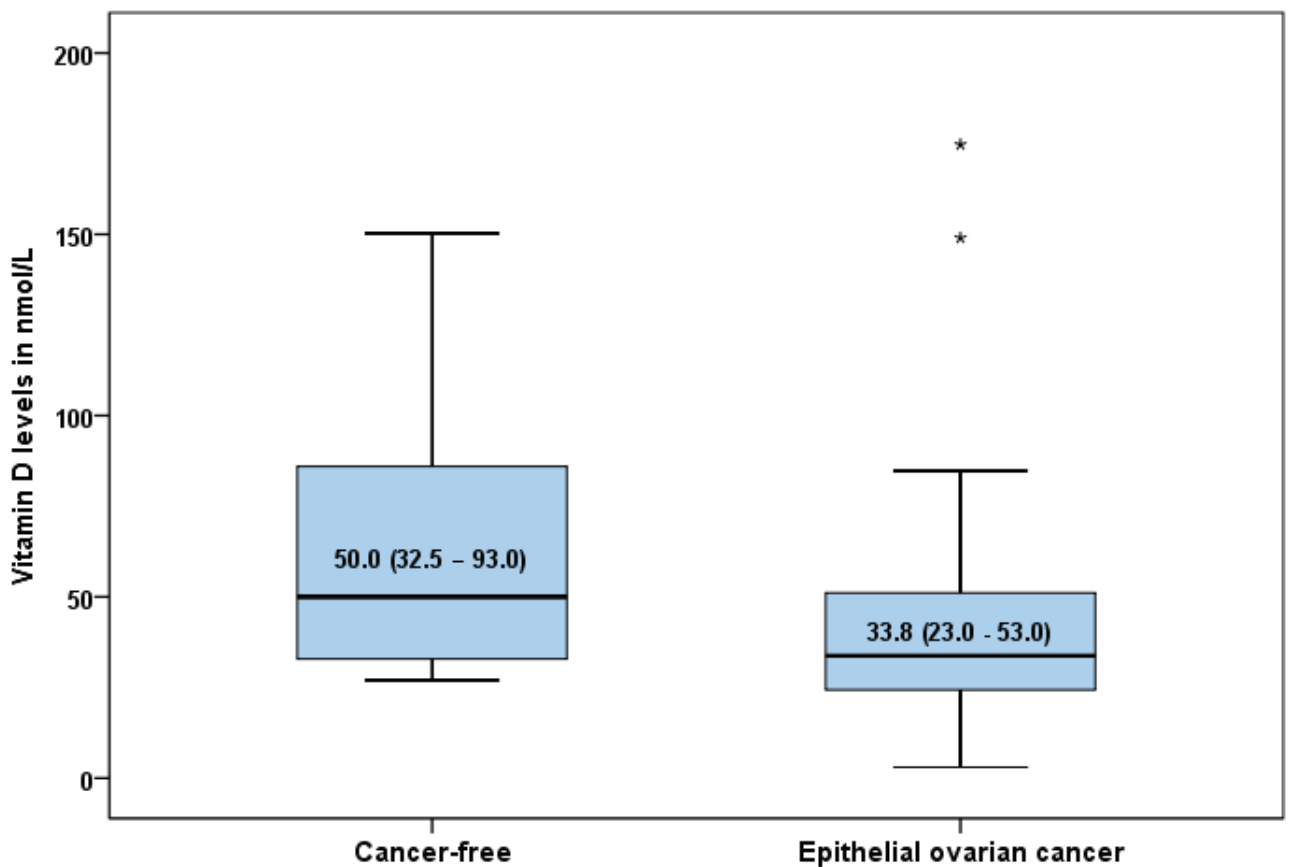

Figure 1. Box plot showing the median serum vitamin D levels in women with epithelial ovarian cancer and those without cancer (50.0 (32.5-93.0) versus $33.8(23.0-53.0) \mathrm{nmol} / \mathrm{l} ; p=0.005)$.

Table 2. Characteristics of women with epithelial ovarian cancer $(n=35)$.

\begin{tabular}{|l|l|}
\hline Characteristics & Number (\%) \\
\hline Histological types of EOC & $28(80.0)$ \\
\hline Serous cystadenocarcinoma & $4(11.4)$ \\
\hline Mucinous cystadenocarcinoma & $2(5.7)$ \\
\hline Endometrioid & $1(2.9)$ \\
\hline Malignant Brenner & $3(8.6)$ \\
\hline Serum CA 125 Level & $32(91.4)$ \\
\hline Normal levels (0-35 U/ml) & \multicolumn{2}{|l|}{} \\
\hline Abnormal levels (>35 U/ml) & $2(5.7)$ \\
\hline FIGO stage of the disease & $33(94.3)$ \\
\hline Early stage & \multicolumn{2}{|l|}{} \\
\hline Advanced stage &
\end{tabular}

Abbreviations: EOC, Epithelial ovarian cancer; CA 125, cancer antigen 125; FIGO, International Federation of Gynaecology and Obstetrics; 
Table 3. Univariable logistic regression of risk factors of epithelial ovarian cancer.

\begin{tabular}{|c|c|c|c|}
\hline Variables & OR & $95 \% \mathrm{Cl}$ & $p$-value \\
\hline \multicolumn{4}{|l|}{ Age group (years) } \\
\hline$<50$ & 1.00 & Reference & - \\
\hline$\geq 50$ & 1.41 & $0.55-3.62$ & 0.47 \\
\hline \multicolumn{4}{|l|}{ BMI $\left(\mathrm{kg} / \mathrm{m}^{2}\right)$} \\
\hline$<25$ (underweight/normal) & 1.00 & Reference & - \\
\hline 25-29 (overweight) & 0.73 & $0.24-2.18$ & 0.57 \\
\hline$\geq 30$ (Obese) & 0.63 & $0.19-2.13$ & 0.46 \\
\hline \multicolumn{4}{|l|}{ Parity } \\
\hline 0 & 1.00 & Reference & - \\
\hline $1-4$ & 0.87 & $0.23-3.30$ & 0.84 \\
\hline$>4$ & 0.65 & $0.14-3.04$ & 0.58 \\
\hline \multicolumn{4}{|l|}{ Family history of cancer } \\
\hline No & 1.00 & Reference & - \\
\hline Yes & 4.89 & $0.96-24.97$ & 0.06 \\
\hline \multicolumn{4}{|l|}{ Alcohol use } \\
\hline No & 1.00 & Reference & - \\
\hline Yes & 3.19 & $0.32-32.24$ & 0.33 \\
\hline \multicolumn{4}{|l|}{ Educational qualification } \\
\hline At least primary education & 1.00 & Reference & - \\
\hline At least secondary education & 0.56 & $0.16-1.93$ & 0.36 \\
\hline \multicolumn{4}{|l|}{ Skin colour } \\
\hline Light & 1.00 & Reference & - \\
\hline Black & 0.58 & $0.14-2.37$ & 0.45 \\
\hline Dark brown & 0.44 & $0.14-1.38$ & 0.16 \\
\hline \multicolumn{4}{|l|}{ Coexisting morbidity } \\
\hline No & 1.00 & Reference & - \\
\hline Yes & 2.30 & $0.62-8.48$ & 0.21 \\
\hline \multicolumn{4}{|l|}{ Menopausal status } \\
\hline Premenopausal & 1.00 & Reference & - \\
\hline Postmenopausal & 1.27 & $0.49-3.31$ & 0.63 \\
\hline \multicolumn{4}{|l|}{ Vitamin D category } \\
\hline Normal ( $\geq 75 \mathrm{nmol} / \mathrm{l})$ & 1.00 & Reference & - \\
\hline Deficiency (<75 nmol/l) & 2.75 & $0.84-9.00$ & 0.09 \\
\hline Serum vitamin D levels per $10 \mathrm{nmol} / \mathrm{l}$ & 0.96 & $0.93-0.99$ & 0.04 \\
\hline
\end{tabular}

Abbreviations: BMI, body mass index (calculated as weight in kilograms divided by the square of height in metres); OR, crude odds ratio. 
Table 4. Multivariable conditional logistic regression of the major risk factors of epithelial ovarian cancer.

\begin{tabular}{|l|l|l|l|}
\hline Variables & OR & $(95 \% \mathrm{Cl})$ & $p$-value \\
\hline Serum vitamin D levels per $10 \mathrm{nmol} / \mathrm{L}$ & 0.99 & $0.97-1.00$ & 0.06 \\
\hline Age (years) & & & \\
\hline$<50$ & 1.00 & Reference & - \\
\hline$\geq 50$ & 1.56 & $0.54-4.51$ & 0.42 \\
\hline Family history of cancer & & & \\
\hline No & 1.00 & Reference & - \\
\hline Yes & 5.88 & $1.05-32.94$ & 0.04 \\
\hline Skin colour & & & \\
\hline Light & 1.00 & Reference & - \\
\hline Black & 0.47 & $0.10-2.18$ & 0.34 \\
\hline Dark brown & 0.37 & $0.11-1.31$ & 0.12 \\
\hline
\end{tabular}

Abbreviations: $\mathrm{Cl}$, confidence interval; OR, adjusted odds ratio.

The major limitation of this study was the difficulty in extracting reliable information on the intake of vitamin D-rich diets and vitamin D supplements from some of the study participants, and this might have had an indirect influence on the findings of this study. There is unrestricted access to medications in the country, and most of the people use varieties of supplements purchased over the counter, which may contain different elements including vitamin D. In addition, the study was conducted in the Lagos metropolis inhabited predominantly by well-educated populace within the middle-to-upper socioeconomic status who are expected to take diets rich in most of the nutrients including vitamin D. Moreover, few women in this study had circulating vitamin D levels above $75 \mathrm{nmol} / \mathrm{L}(22.5 \%)$, and thus, we cannot rule out the possibility that women with very high $25(\mathrm{OH}) \mathrm{D}$ levels may have a reduced risk of EOC. Finally, the study design type may make it difficult to confirm temporality or causality of the vitamin $D$ exposure as the association observed may be due to changes in the circulating vitamin $D$ levels before the disease due to lifestyle or as a result of prolonged confinement due to illness. Nonetheless, this study was an exploratory and hypothesis-generating project, to serve as the basis for the conduct of a more robust study in the near future. The strength of this study is that it is the first of such, to the best of authors' knowledge, which will examine the association between low circulating vitamin $D$ levels and the risk of epithelial ovarian cancer amongst women in an Sub-Saharan African region.

\section{Conclusions}

Overall, we observed no clear association between serum vitamin D deficiency and ovarian cancer risk in this study. A better assessment of sun exposure in the future as well as better dietary compositional data may help to clarify whether the association between vitamin $\mathrm{D}$ and epithelial ovarian cancer actually exists. Therefore, future large prospective longitudinal studies are recommended to further examine this relationship and then evaluate the possible need for vitamin D supplementation in women with an increased or familial risk of epithelial ovarian cancer in Nigeria.

\section{Conflicts of interest}

The authors declare that they have no conflicts of interest. 


\section{Acknowledgments and funding}

KSO acknowledges the partial time protection afforded him through the HBNU Fellowship supported by the Fogarty International Centre and National Institute of Mental Health, of the National Institutes of Health under Award Number D43TW010543 and Conquer Cancer International Innovation Grant under Project ID 16576. GO acknowledges the Medical Education Partnership in Nigeria for the award of a training mentor/mentee fellowship under award number R24TW008878 and the funding through the GSK/DELTAS Africa Initiatives Grant No. 107754/z/15/z-DELTAS Africa SACCAB to study for a PhD in Public health (Biostatistics) at the University of Witwatersrand. The views expressed in this publication are those of the authors and not necessarily those of the funders. The content is solely the responsibility of the authors and does not necessarily represent the official views of the National Institutes of Health or any other funding bodies.

\section{References}

1. Anorlu RI, Obodo K, and Makwe CC (2010) Cancer mortality among patients admitted to gynaecological wards at Lagos University Teaching Hospital, Nigeria Intern J Gynecol Obstet 110(3) 268-269 https://doi.org/10.1016/j.ijgo.2010.03.038

2. Okunade KS, Okunola $\mathrm{H}$, and Okunowo AA, et al (2016) A five-year review of ovarian cancer at a tertiary institution in Lagos, SouthWest, Nigeria Niger J Gen Pract 14(2) 23-27 https://doi.org/10.4103/1118-4647.187901

3. Zayyan MS, Ahmed SA, and Oguntayo AO, et al (2017) Epidemiology of ovarian cancers in Zaria, Northern Nigeria: a 10-year study Int J Womens Health 9 855-860 https://doi.org/10.2147/IJWH.S130340 PMID: 29200893 PMCID: 5702529

4. Iyoke CA, Ugwu GO, and Ezugwu EC, et al (2013) Incidence, pattern and management of ovarian cancer at a tertiary medical center in Enugu, South East Nigeria Ann Med Health Sci Res 3 417-421 https://doi.org/10.4103/2141-9248.117947 PMID: 24116325 PMCID: 3793451

5. Ferley J, Soerjomataram I, and Dikshit R, et al (2015) Cancer incidence and mortality worldwide: Sources, methods and major patterns in GLOBOCAN 2012 Int J Cancer 136(5) E359-E386 https://doi.org/10.1002/ijc.29210

6. Thompson D and Easton DF (2002) Cancer incidence in BRCA 1 mutation carriers J Natl Cancer Inst 94 1358-1365 https://doi. org/10.1093/jnci/94.18.1358 PMID: 12237281

7. Stratton JF, Pharoah $\mathrm{P}$, and Smith SK, et al (1998) A systematic review and meta- analysis of family history and risk of ovarian cancer Br J Obstet Gynaecol 105 493-499 https://doi.org/10.1111/j.1471-0528.1998.tb10148.x PMID: 9637117

8. Schildkraut JM, Cooper GS, and Halabi S, et al (2001) Age at natural menopause and the risk of epithelial ovarian cancer Obstet Gynecol 98(1) 85-90 PMID: 11430962

9. Brekelmans CT (2002) Risk factors and risk reduction of breast and ovarian cancer Curr Opin Obstet Gynecol 15(1) 63-68 https://doi. org/10.1097/00001703-200302000-00010

10. Walker GR, Schlesselman JJ, and Ness RB (2002) Family history of cancer, oral contraceptive use, and ovarian cancer risk Am J Obstet Gynecol 186 8-14 https://doi.org/10.1067/mob.2002.118657 PMID: 11810077

11. Bertone ER, Newcomb PA, and Willett WC, et al (2002) Recreational physical activity and ovarian cancer in a population-based casecontrol study Int J Cancer 99(3) 431-436 https://doi.org/10.1002/ijc.10365 PMID: 11992414

12. Saad AF, Hu W, and Sood AK (2010) Microenvironment and pathogenesis of epithelial ovarian cancer Horm Cancer 1(6) 277-290 https://doi.org/10.1007/s12672-010-0054-2

13. Zitterman A and Gunmmert JF (2010) Non-classical vitamin D action Nutrients 2 408-425 https://doi.org/10.3390/nu2040408

14. Wacker M and Holick MF (2013) Vitamin D effects on skeletal and extraskeletal health and the need for supplementation Nutrients 5 111-148 https://doi.org/10.3390/nu5010111 PMID: 23306192 PMCID: 3571641 
15. Holick MF (2007) Vitamin D deficiency N Engl J Med 357(3) 266-281 https://doi.org/10.1056/NEJMra070553 PMID: 17634462

16. Moan J, Porojnicu AC, and Dahlback A, et al (2008) Addressing the health benefits and risks, involving vitamin D or skin cancer, of increased sun exposure Proc Natl Acad Sci USA 105(2) 668-673 https://doi.org/10.1073/pnas.0710615105 PMID: 18180454 PMCID: 2206594

17. Arslan AA, Clendenen TV, and Koenig KL, et al (2009) Circulating vitamin D and risk of epithelial ovarian cancer J Oncol 6724928

18. Genkinger JM, Hunter DJ, and Spiegelman D, et al (2006) Dairy products and ovarian cancer: a pooled analysis of 12 cohort studies Cancer Epidemiol Biomarkers Prev 15(2) 364-372 https://doi.org/10.1158/1055-9965.EPI-05-0484 PMID: 16492930

19. Larsson SC, Orsini N, and Wolk A (2006) Milk, milk products and lactose intake and ovarian cancer risk: a meta-analysis of epidemiological studies Int J Cancer 118(2) 431-441 https://doi.org/10.1002/ijc.21305

20. Tworoger SS, Lee MI, and Buring JE, et al (2007) Plasma 25-hydroxyvitaminD and 1,25-Dihydroxyvitamin D and risk of incident ovarian cancer Cancer Epidemiol Biomarkers Prev 16(4) 783-788 https://doi.org/10.1158/1055-9965.EPI-06-0981 PMID: 17416771

21. Bakhru A, Mallinger JB, and Buckanovich RJ, et al (2010) Casting light on 25-Hydroxyvitamin D deficiency in ovarian cancer: a study from NHANES Gynecol Oncol 119(2) 314-318 https://doi.org/10.1016/j.ygyno.2010.07.006 PMID: 20684976

22. Webb PM, Fazio AD, and Protani MM, et al (2015) Circulating 25-hydroxyvitamin D and survival in women with ovarian cancer Am J Clin Nutr 102(1) 109-114 https://doi.org/10.3945/ajcn.114.102681 PMID: 25971716

23. Pandian R, Pandian J, and Elias R Clinical Importance of "Bioavailable" Vitamin D Development and Analytical Validation of Bioavailable 25 Hydroxy Vitamin D assay American Assiociation for Clinical Chemistry, Houston, TX, 28 July-1 August 2013

24. Lefkowitz ES and Garland CF (1994) Sunlight, vitamin D, and ovarian cancer mortality rates in US women Int J Epidemiol 23(6) 11331136 https://doi.org/10.1093/ije/23.6.1133 PMID: 7721513

25. Grant WB (2002) An estimate of premature cancer mortality in the U.S. due to inadequate doses of solar ultraviolet-B radiation Cancer 94(6) 1867-1875 https://doi.org/10.1002/cncr.10427 PMID: 11920550

26. Freedman DM, Dosemeci M, and McGlynn K (2002) Sunlight and mortality from breast, ovarian, colon, prostate, and non-melanoma skin cancer: a composite death certificate-based case-control study Occup Environ Med 59(4) 257-262 https://doi.org/10.1136/ oem.59.4.257 PMID: 11934953 PMCID: 1740270

27. Garland CF, Mohr SB, and Gorham ED, et al (2006) Role of ultraviolet B irradiance and vitamin D in prevention of ovarian cancer Am J Prev Med 31(6) 512-514 https://doi.org/10.1016/j.amepre.2006.08.018 PMID: 17169713

28. Zheng W, Danforth KN, and Tworoger SS, et al (2010) Circulating 25-hydroxyvitamin d and risk of epithelial ovarian cancer: cohort consortium vitamin d pooling project of rarer cancers Am J Epidemiol 172(1) 70-80 https://doi.org/10.1093/aje/kwq118 PMID: 20562186 PMCID: $\underline{2892541}$

29. Toriola AT, Surcel HM, and Agborsangaya C, et al (2010) Serum 25-hydroxyvitamin D and the risk of ovarian cancer Eur J Cancer 46(2) 364-369 https://doi.org/10.1016/j.ejca.2009.08.002

30. Deluca H (2004) Overview of general physiologic features and function of vitamin D Am J Clin Nutr 80 1689-1696 https://doi. org/10.1093/ajcn/80.6.1689S

31. Holick MF (2004) Sunlight and vitamin D for bone health and prevention of autoimmune disease, cancer and cardiovascular disease $\mathrm{Am}$ J Clin Nutr 80 1678-1688 https://doi.org/10.1093/ajcn/80.6.1678S

32. Antonio A, Pharaoh PDP, and Narod S, et al (2003) Average risks of breast and ovarian cancer associated with BRCA1 or BRCA2 mutations detected in case series unselected for family history: a combined analysis of 22 studies Am J Human Genet 72(5) 1117-1130 https://doi.org/10.1086/375033 